Int. J. Curr. Res. Med. Sci. (2021). 7(1): 1-5

\begin{tabular}{|c|c|c|}
\hline & $\begin{array}{c}\text { International Journal of Current Research in } \\
\text { Medical Sciences } \\
\text { ISSN: 2454-5716 } \\
\text { (A Peer Reviewed, Indexed and Open Access Journal) } \\
\text { WWW.ijcrims.com }\end{array}$ \\
\hline
\end{tabular}

\title{
Central nervous system Tuberculomas in a patient with disseminated multi-drug resistant tuberculosis; A case report
}

\author{
Harveen Kaur $^{1 *}$, Dilbag Singh ${ }^{1}$, Amritpal Kaur ${ }^{1}$, N.C Kajal', Mukul Sharma ${ }^{3}$ \\ ${ }^{1}$ Junior resident, Department of Pulmonary Medicine, Government Medical College, Amritsar Punjab, India \\ ${ }^{2}$ Professor, Department of pulmonary Medicine, Government Medical College, Amritsar Punjab, India \\ ${ }^{3}$ Junior resident, Department of CTVS, Apollo Hospital, Hyderabad, Telangana, India \\ *Corresponding Author E-mail: hk_94basra@ymail.com
}

\begin{abstract}
Tuberculosis (TB) is one of the leading causes of death worldwide, particularly in low- and middle-income countries. Although Mycobacterium tuberculosis can involve any organ, most commonly the lung, central nervous system (CNS) tuberculosis is the most devastating form of the disease. Tuberculoma is the most common parenchymal lesion in CNS tuberculosis which could be found in any portion of the intracranial space. The global rates and numbers of drug resistant TB are rising. With increasing globalization, the spread of drug-resistant strains of TB has become a mounting global public health concern. We present a case of 27-year-old male with disseminated multi-drug resistant (MDR) TB who presented with neurological symptoms and multiple CNS Tuberculomas. The patient was started on regimen for Multi-drug resistant tuberculosis (MDR-TB), which allowed the serial resolution of intracranial tuberculomas.
\end{abstract}

Keywords: Tuberculosis (TB), Multi-drug resistant (MDR) TB, Central nervous system (CNS) TB, Tuberculoma, MRI brain

\section{Introduction}

Tuberculosis (TB) remains one of the top ten causes of death worldwide. In 2015, an estimated 10.4 million people worldwide developed TB and 1.8 million died; over $95 \%$ of these deaths occurred in low- and middle-income countries. ${ }^{1}$ Resistance to standard anti-TB regimens is becoming widespread in the form of multidrugresistant (MDR) and extensively drug-resistant (XDR) tuberculosis. MDR-TB is defined as resistance to isoniazid and rifampin, two of the most potent first-line TB drugs; XDR-TB is resistance to isoniazid, rifampin, the fluoroquinolones, as well as any of the second line injectable agents. ${ }^{1}$ Globally, MDR-TB and XDR-TB notifications are both increasing. There were an estimated 480,000 people globally who developed MDR-TB in 2015; the largest numbers of these cases were reported in India, China, and Russia, and $9.5 \%$ of MDR cases met the definition of XDR-TB. ${ }^{1}$ While TB most commonly infects the lungs, TB can occur in any organ in the body, including the central nervous 
system (CNS). CNS TB occurs in approximately $1 \%$ of patients with $\mathrm{TB}$ disease and is associated with major morbidity and mortality. The type of lesions that result from discharge of the bacilli into the cerebrospinal fluid (CSF) depends on the virulence of the bacteria and the immune resistance of the host. The brain lesion also originates from hematogenous spread from the lungs, and when there is a sizeable inoculation with inadequate cell-mediated immunity, the parenchymal lesion may develop into a tuberculoma.

Here we present a case of27 year old immunocompetent male with MDR-PulmonaryTB with neurological symptoms and MRI Brain showing multiple CNS tuberculomas.

\section{Case Report}

A 27 year old male presented to the hospital with headache, diplopia, cough, low grade fever, loss of appetite, and weight loss since past 3 months. Patient had no significant past and personal history. Patient's father had history of pulmonary tuberculosis. Neurologic examination showed a fully alert, oriented, and cooperative patient. Complete blood count (CBC) and other routine blood tests (complete metabolic panel including liver function tests, erythrocyte sedimentationrate (ESR), C-reactive protein (CRP), thyroid stimulating hormone (TSH) were within normal limits. HIV serology were nonreactive. Chest radiography revealed bilateral infiltrates, predominantly involving both upper zones. Keeping the possibility of pulmonary tuberculosis, bronchoscopy was performed as the patient was not producing sputum. Drug susceptibility testing from bronchial aspirate revealed resistance to rifampicin and isoniazid. MRI brain showed tuberculomas as multiple 3$4 \mathrm{~mm}$ sized ring enhancing lesions involving both cerebral hemisphere, pons and left cerebellar hemisphere with meningeal enhancement involving right post-central sulcus. Ophthalmologic assessment revealed CDR 0.41 in right and left eye with normal visual acuity. Based on these results, patient was started on longer all oral MDR regimen comprising of bedaquiline, levofloxacin, linezolid, clofazimine and cycloserine along with pyridoxine.

A repeat MRI done after 2 months revealed a significant reduction in the size of lesions as multiple small 1-2mm well defined seen in left frontal, bilateral parieto-temporal, left parasaggital, left occipital and pons region with rest of the brain parenchyma showing normal signal intensity.

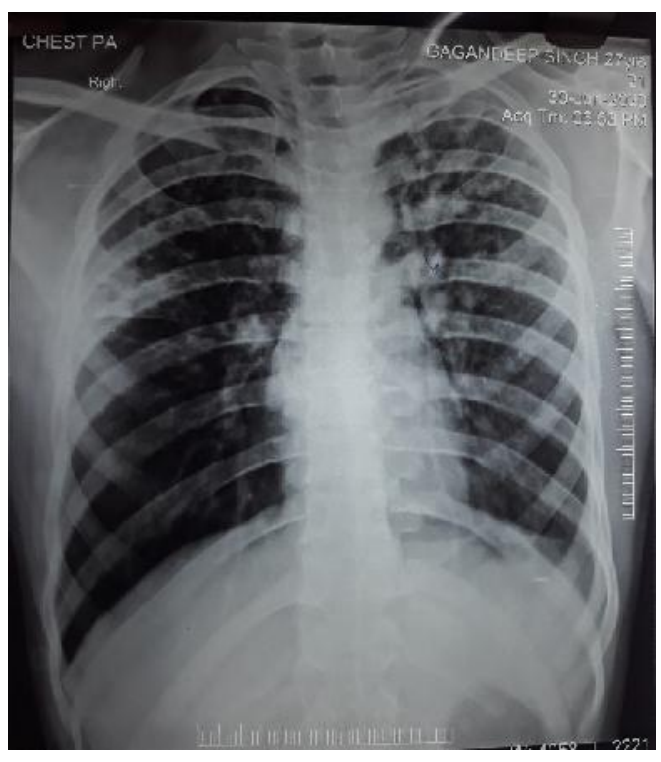

Figure 1 Chest X-Ray PA view of patient showing bilateral infiltrates, predominantly involving both upper zones. 
Int. J. Curr. Res. Med. Sci. (2021). 7(1): 1-5

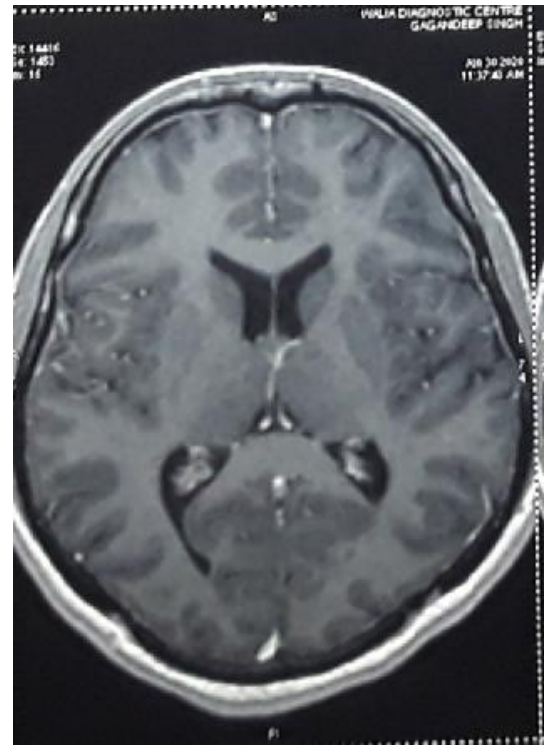

Figure 2

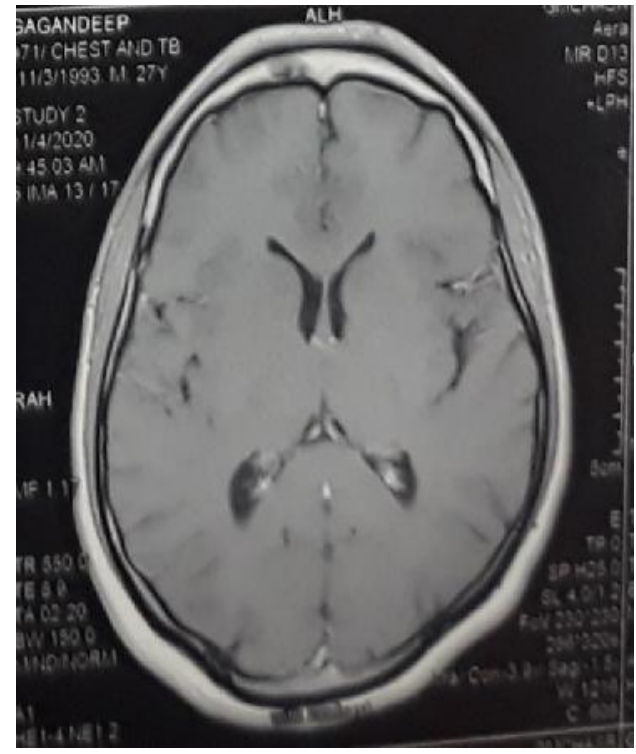

Figure 3

Serial MRI brain shows marked reduction in size and number of lesions compared with pre-treatment, with diminution of edema around lesion.

\section{Discussion}

The incidence of tuberculosis has increased in recent years. In addition, the occurrence of outbreaks caused by multidrug-resistant $\mathrm{M}$. tuberculosis has become increasingly a matter of concern. Extrapulmonary MDR-TB is treated with the same strategy involving the same regimen and duration as pulmonary MDRTB. ${ }^{2}$ Central nervous system tuberculosis is a rare entity affecting 0.5 to $2 \%$ of patients with systemic tuberculosis. The majority of the lesions are intracranial. On CT or MR imaging, tuberculomas appear as low- or high-density round or lobulated masses with irregular walls showing homogenous enhancement after contrast administration. They may be solitary or multiple and have a predilection to occur in the frontal and parietal lobes. The authors emphasize the difficulty in differentiating these lesions from those of other etiologies. ${ }^{3}$ Contrast-enhanced MR scanning is superior to CT Scans in assessing meningeal and parenchymal abnormalities. Follow-up MR studies are useful in monitoring the response to medical treatment. However, with continuation of antituberculous therapy, eventual resolution of the tuberculoma usually occurs. ${ }^{4,5}$
The prevalence of MDR-TB is increasing in number in recent years because of increased awareness of the disease, increased access to culture and drug sensitivity testing, and earlier suspicion of MDR-TB cases among previously treated patients. So, it is expected that MDR-TB affecting extrapulmonary sites will increase in the near future. ${ }^{6}$ Hence, there is an urgent need for increasing awareness of the physicians to such presentation.

The patient discussed here was diagnosed as a case of MDR pulmonary tuberculosis having multiple CNS tuberculomas with neurological symptoms. Given the mortality of CNS tuberculoma without treatment is estimated between $35-85 \%$, and the precarious location of the lesion in question and its likely activity, we concluded that there was sufficient urgency to treat this patient empirically and aggressively. ${ }^{7,8}$ In patients managed with appropriate therapy, the prognosis for neurologic improvement is good. ${ }^{9}$ Patients with MDR-TB frequently have advanced disease associated with thick-walled cavities and chronic lung lesions that can be difficult for antimicrobial drugs to penetrate. ${ }^{10}$ 
Our patient had unusual disseminated manifestations in the CNS without typical cavernous lesions in the lung. Based on drug susceptibility testing, the patient was resistant to rifampicin and isoniazid. $\mathrm{He}$ was initiated on MDR regimen comprising levofloxacin, linezolid, cycloserine, clofazimine and bedaquiline as per RNTCP/ PMDT guidelines. Bedaquiline inhibits mycobacterial ATP synthase and has been approved by the United States Food and Drug Administration (FDA) and the World Health Organization for use in drug-resistant $\mathrm{TB} .{ }^{11} \mathrm{He}$ improved clinically and radiographically after treatment initiation.

\section{Conclusion}

We have reported this case to emphasize the increasing incidence of extrapulmonary MDR$\mathrm{TB}$, the importance of rapid and reliable diagnosis, and to create an awareness for early suspicion. Further research is urgently needed to know the prevalence, diagnostic procedure, effective regimen and duration of treatment, monitoring procedure and outcome of the patients of MDR extrapulmonary TB. This case illustrates the greater issue of increasing $\mathrm{TB}$ and drug resistant TB as a global public health emergency. In an era of globalization and continuing disparities of health and resources that underlie the spread of TB, we acknowledge the need to support global TB efforts to prevent the spread of drug-resistant $\mathrm{TB}$, and to maintain $\mathrm{TB}$ expertise and patient supportive services.

\section{References}

1. World Health Organization Tuberculosis fact sheet 201619 October http://www.who.int/mediacentre/factsheets/fs 104/en/ (2016)

2. Guidelines for the programmatic management of drug-resistant tuberculosis. Geneva, World Health Organization, Emergency Update, 2008 (WHO/HTM/TB/2008.402).

3. Dube MP Holtom PD, Larsen RA Tuberculous meningitis in patients with and without human immunodeficiency virus infection. Am J Med 1992; 93: j20- 524.

4. B. D. Ku and S. Yoo, "Extensive meningeal and prenchymal calcified tuberculoma as long-term residual sequelae of tuberculous meningitis," Neurology India, vol. 57, no. 4, pp. 521-522, 2009.

5. B. Afghani and J. M. Lieberman, "Paradoxical enlargement or development of intracranial tuberculomas during therapy: case report and review," Clinical Infectious Diseases, vol. 19, no. 6, pp. 1092-1099, 1994.

6. Rawat J, Sindhwani G, Dua R. Primary multidrug resistant tubercular lymphadenitis in an HIV infected patient. Indian J Tuberc 2009; 56:157-9.

7. S Gulsen, $\mathrm{H}$ CanerTuberculoma in the medulla oblongata and medulla spinalis: two case reports Balkan Med J, 30 (4) (2013), pp. $442-445$

8. G Thwaites, M FIsher, C Hemingway, G Scot, T Solomon, J Innes. British infection society guidelines for the diagnosis and treatment of tuberculosis of the central nervous system in adults and children J Infect, 59 (2009), pp. 167-187 
9. Rhoton EL, Ballinger WE, Quisling R, Sypert GW. Intramedullary spinal tuberculoma. Neurosurgery 1988; 4:733-736.

10. Mukherjee JS, Rich ML, Socci AR, et al. Programmes and principles in treatment of multidrug-resistant tuberculosis. Lancet 2004; 363: 474-481.

11. Bedaquiline: drug information [Internet]. 2017 [cited 4 April 2017].

\begin{tabular}{|c|l|}
\hline \multicolumn{2}{|c|}{ Access this Article in Online } \\
\hline Quick Response Code & Website: \\
& Www.ijcrims.com \\
\hline & \\
\hline
\end{tabular}

How to cite this article:

Harveen Kaur, Dilbag Singh, Amritpal Kaur, N.C Kajal, Mukul Sharma. (2021). Central nervous system Tuberculomas in a patient with disseminated multi-drug resistant tuberculosis; A case report. Int. J. Curr. Res. Med. Sci. 7(1): 1-5.

DOI: http://dx.doi.org/10.22192/ijcrms.2021.07.01.001 